\title{
Effect of a single dose of esmolol on the bispectral index to endotracheal intubation during desflurane anesthesia
}

\author{
Eun Mi Choi ${ }^{1}$, Kyeong Tae Min ${ }^{2}$, Jeong Rim Lee ${ }^{2}$, Tai Kyung Lee ${ }^{2}$, and Seung Ho Choi ${ }^{2}$ \\ ${ }^{1}$ Department of Anesthesiology and Pain Medicine, Kangnam Sacred Heart Hospital, Hallym University College of Medicine, \\ ${ }^{2}$ Department of Anesthesiology and Pain Medicine, Anesthesia and Pain Research Institute, Yonsei University College of Medicine, \\ Seoul, Korea
}

Background: In this prospective, randomized, double-blind, placebo-controlled trial, we investigated the effect of a single dose of esmolol on the bispectral index (BIS) to endotracheal intubation during desflurane anesthesia.

Methods: After induction of anesthesia, 60 patients were mask-ventilated with desflurane (end-tidal 1 minimum alveolar concentration) for $5 \mathrm{~min}$ and then received either normal saline, esmolol $0.5 \mathrm{or} 1 \mathrm{mg} / \mathrm{kg}, 1 \mathrm{~min}$ prior to intubation (control, esmolol-0.5 and esmolol-1 groups, $n=20$ /group). BIS, mean arterial pressure, and heart rate were measured prior to anesthesia induction and esmolol administration, immediately preceding intubation (time point 0 ), and every minute for $5 \mathrm{~min}$ after intubation (time point 1 to 5 ). At time point 0,1 and 5, $5 \mathrm{ml}$ of arterial blood was taken to measure plasma concentrations of norepinephrine and epinephrine.

Results: BIS increased significantly at 1 min after intubation when compared with pre-intubation values in all groups. Both mean arterial pressure and heart rate increased significantly $1 \mathrm{~min}$ after intubation when compared with preintubation values for all groups. Plasma epinephrine concentrations did not increase significantly after tracheal intubation in any of the groups. Norepinephrine increased at $1 \mathrm{~min}$ after intubation when compared with the preintubation values in the esmolol groups $(\mathrm{P}<0.05)$.

Conclusions: A single bolus of esmolol was unable to blunt the increase in BIS to endotracheal intubation during desflurane anesthesia. (Korean J Anesthesiol 2013; 64: 420-425)

Key Words: Bispectral index, Desflurane, Esmolol, Intubation, Norepinephrine.

\footnotetext{
Received: July 26, 2012. Revised: September 24, 2012. Accepted: October 11, 2012.

Corresponding author: Seung Ho Choi, M.D., Department of Anesthesiology and Pain Medicine, Anesthesia and Pain Research Institute, Yonsei University College of Medicine, CPO Box 8044, Seoul 120-752, Korea. Tel: 82-2-2228-2429, Fax: 82-2-312-7185, E-mail: csho99@yuhs.ac

(c) This is an open-access article distributed under the terms of the Creative Commons Attribution Non-Commercial License (http:// creativecommons.org/licenses/by-nc/3.0/), which permits unrestricted non-commercial use, distribution, and reproduction in any medium, provided the original work is properly cited.
} 


\section{Introduction}

The central sympathetic adrenergic response, which occurs during endotracheal intubation via an activation of the peripheral nervous system activation, results in an increase in the bispectral index (BIS) [1]. Accordingly, many interventions are used in an attempt to blunt the increase in BIS to endotracheal intubation [2-4]. Esmolol produces a dose-dependent attenuation of this adrenergic response and thus effectively prevents arousal reactions to noxious stimuli [2-5]. In a previous study, a single dose of esmolol $(0.5 \mathrm{mg} / \mathrm{kg})$ blunted the increase in BIS to endotracheal intubation with sevoflurane anesthesia, but not with desflurane anesthesia [5]. Additionally, desflurane is believed to induce greater neurocirculatory excitation - producing larger increases in sympathetic nervous activity, central venous pressure, and plasma norepinephrine (NE) concentrations when compared with other inhaled anesthetics $[6,7]$. To the best of our knowledge, the effect of a single dose of esmolol on the catecholamine response to endotracheal intubation during desflurane anesthesia has not been investigated. As such, we hypothesized that a single dose of esmolol $1 \mathrm{mg} / \mathrm{kg}$, which is a larger dose than that of the previous study [5], might blunt the BIS to endotracheal intubation during desflurane anesthesia. Hence, in this prospective, randomized, double blind, placebocontrolled trial, we evaluate the effect of esmolol on these endpoints.

\section{Materials and Methods}

After receiving an approval from the Institutional Review Board, 60 adult patients were enrolled in the present study. Written informed consent was obtained in all cases, and all subjects had an American Society of Anesthesiologists physical status classification of either I or II, and were between 18 and 65 years of age. Patients with cardiovascular, respiratory, or neurological disease including individuals taking cardiovascular medications or predicted difficulty in endotracheal intubation were excluded from the study. Patients were then randomly divided into 3 groups of 20 by computer-generated sequence, receiving either normal saline (control group), esmolol $0.5 \mathrm{mg} /$ $\mathrm{kg}$ (esmolol-0.5 group), or esmolol $1 \mathrm{mg} / \mathrm{kg}$ (esmolol-1 group).

No premedication was administered in any case. On arrival to the operating room, all patients were monitored by electrocardiography, noninvasive blood pressure, and pulse oximetry. For BIS monitoring, a standard BIS monitor strip (BIS Sensor ${ }^{\circledR}$; Aspect Medical Systems, Newton, MA, USA) was placed on the forehead so that BIS (Model A-2000; Aspect Medical Systems, Newton, MA, USA) could be measured continuously.

The anesthetic technique used here was standardized: first anesthesia was induced with thiopental $5 \mathrm{mg} / \mathrm{kg}$, after which rocuronium $0.6 \mathrm{mg} / \mathrm{kg}$ was administered intravenously to achieve neuromuscular blockade. Patients were manually ventilated to maintain $\mathrm{EtCO}_{2}$ between $35-40 \mathrm{mmHg}$, and desflurane was administered via facemask with a concomitant oxygen flow of $5 \mathrm{~L} / \mathrm{min}$. The concentration of desflurane was then increased until the end-tidal concentration reached 1 minimum alveolar concentration (MAC), and administered for $6 \mathrm{~min}$ prior to endotracheal intubation. A 20-gauge radial artery catheter was inserted for the purpose of continuous arterial pressure monitoring and blood sampling. One minute prior to endotracheal intubation, either normal saline, esmolol 0.5 $\mathrm{mg} / \mathrm{kg}$ or esmolol $1 \mathrm{mg} / \mathrm{kg}$ was administered intravenously to patients in a double-blinded manner. All syringes containing the study drug (same volume) were prepared by an anesthesiologist not involved in the study. One minute after the administration of the normal saline or esmolol, endotracheal intubation was performed by an experienced anesthesiologist, also not involved in the study and was accomplished in less than 20 seconds. After endotracheal intubation, the lungs were ventilated with $50 \%$ air in oxygen and a 1 MAC end-tidal concentration of desflurane. No additional drugs were administered to any subjects, and no surgical procedures were performed during the study period. BIS, mean arterial blood pressure (MAP) and heart rate (HR) were measured prior to induction of anesthesia (awake), before esmolol injection (time point -1), immediately before intubation (time point 0 ), and each minute for $5 \mathrm{~min}$ after endotracheal intubation (time points 1 to 5 ). If systolic blood pressure decreased below $90 \mathrm{mmHg}$, or if the heart rate decreased below 50 beats/min, ephedrine $4 \mathrm{mg}$ was administered intravenously.

Arterial blood samplings $(5 \mathrm{ml})$ were also collected at time points 0,1 , and 5 . Within 20 min after collection, these samples underwent plasma separation by centrifuge at 3,000 r.p.m. for 7 min, then were frozen to $-70^{\circ} \mathrm{C}$. All plasma epinephrine (EPI) and NE concentrations were measured by high-pressure liquid chromatography, with detection limits for EPI and NE of 20 $\mathrm{pg} / \mathrm{ml}$ and $20 \mathrm{pg} / \mathrm{ml}$, respectively. The coefficients of variation were $3.49-6.14 \%$ for EPI and $1.51-6.33 \%$ for NE within runs, and $4.42-6.96 \%$ and $3.92-7.46 \%$ between runs. Samples with values less than the limits of detection were considered as having concentrations just below the limit of detection.

Based on a previous study [5], the sample size required to detect a mean difference of 10 in BIS with a SD of 10 and a power of $80 \%$ at an alpha level of 0.05 was 17 patients. In order to compensate for a dropout rate of $10 \%, 20$ patients for each group were enrolled. All data were expressed as mean \pm SD. SPSS version 15.0 (SPSS Inc., Chicago, IL, USA) was used for all statistical analyses. Specifically, the chi-square test was used to compare sex ratios between the three groups, while comparisons of age, weight, height, BIS, MAP, HR, and catecholamine values 


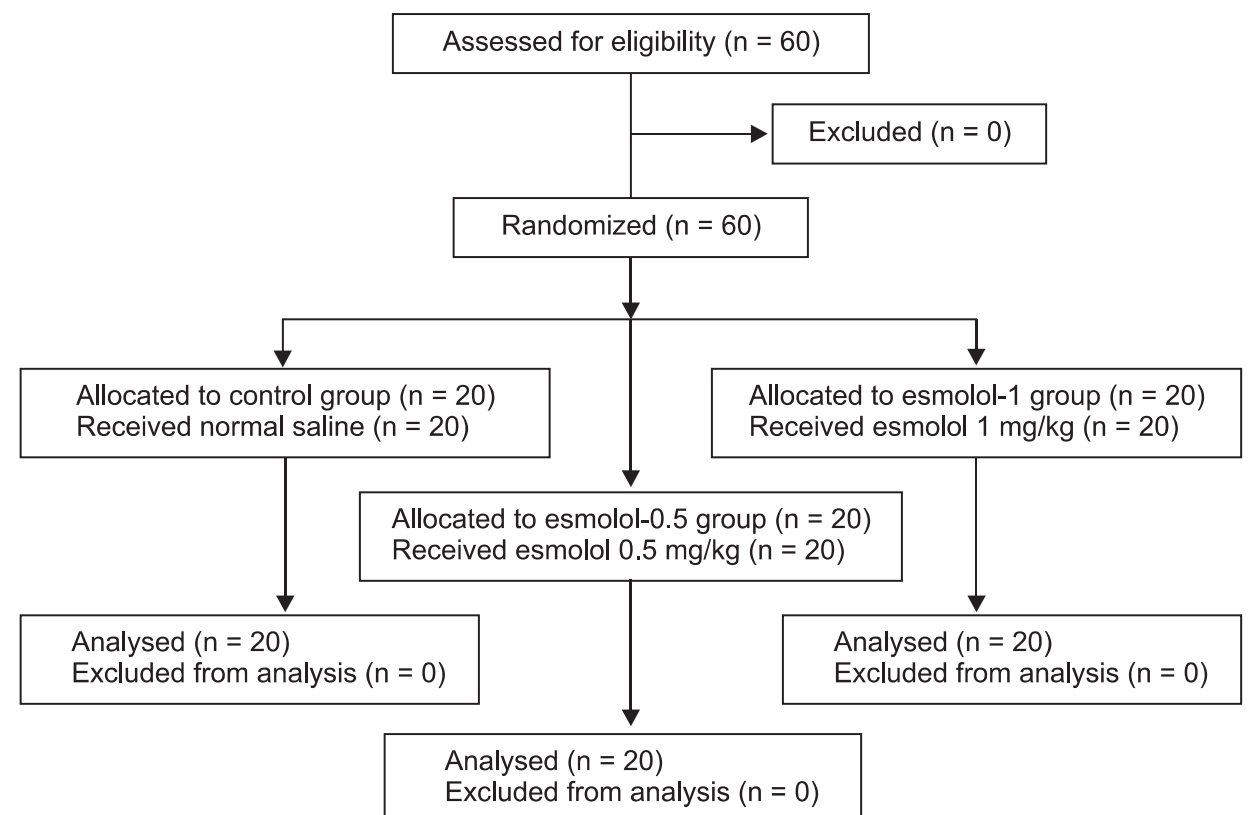

Fig. 1. Flow diagram of the randomized, controlled trial. were assessed using a one-way analysis of variance (ANOVA) with Bonferroni test. Value comparisons for each measurement were also performed using repeated measures of ANOVA. All significant results were also analyzed via Tukey's post hoc test to determine whether time points produced values significantly different from preintubation. A P value $<0.05$ was considered as statistically significant.

\section{Results}

Sixty patients were enrolled in this study and none were excluded or failed to complete (Fig. 1). In total, data from 60 patients were analyzed, with no differences observed in any of the demographic variables (Table 1). Additionally, no patient developed systolic blood pressure $<90 \mathrm{mmHg}$ or $\mathrm{HR}<50$ beats/min during the study period.

The changes in BIS, MAP, and HR during the study period are shown in Fig. 2. At baseline, BIS values were between 95 and 99, and subsequently decreased during anesthesia induction. No intergroup differences were observed in BIS before esmolol administration. At 1 min after intubation, BIS increased significantly in all groups when compared with preintubation ( $34 \pm 6$ to $49 \pm 12$ vs. $36 \pm 5$ to $49 \pm 9$ vs. $36 \pm 5$ to $41 \pm 5 ; \mathrm{P}<0.0001$ vs. $\mathrm{P}<0.0001$ vs. $\mathrm{P}=0.0001$, respectively). However, the BIS value at this time point of the esmolol-1 group was significantly lower than those of the other groups (P $=0.01$ ) (Fig. 2A). The MAP did not differ among groups prior to endotracheal intubation, while the HR also did not differ among groups before the esmolol administration. Both the MAP and HR increased significantly at $1 \mathrm{~min}$ after intubation
Table 1. Demographic Data

\begin{tabular}{lccc}
\hline & Control & Esmolol-0.5 & Esmolol-1 \\
\hline Sex (M/F) & $10 / 10$ & $10 / 10$ & $10 / 10$ \\
Age (yr) & $41.7 \pm 10.8$ & $40.8 \pm 10.9$ & $41.8 \pm 11$ \\
Weight (kg) & $64.2 \pm 10.3$ & $62.9 \pm 10.6$ & $61.8 \pm 10.3$ \\
Height (cm) & $165.4 \pm 8.5$ & $165.5 \pm 11$ & $165.1 \pm 7.2$ \\
\hline
\end{tabular}

Values are presented as mean \pm SD and the number of patients. No statistically significant differences were observed among the three groups. Control: group with receiving normal saline, Esmolol-0.5: group with receiving esmolol $0.5 \mathrm{mg} / \mathrm{kg}$, Esmolol-1: group with receiving esmolol $1 \mathrm{mg} / \mathrm{kg}$ at $1 \mathrm{~min}$ prior to intubation.

in all groups when compared with the preintubation values. The MAP did not differ among three groups after intubation, whereas the HR during the time period 1 to 5 min after intubation in both the esmolol-0.5 and esmolol-1 groups was lower significantly than in the control groups $(\mathrm{P}<0.0001)$ (Fig. $2 \mathrm{~B}$ and $2 \mathrm{C}$ ). Plasma EPI and NE concentrations during the study period are shown in Fig. 3. EPI did not increase significantly after endotracheal intubation in any of the groups, whereas NE increased 1 min after intubation when compared with the preintubation measurements in the esmolol groups $(\mathrm{P}<0.05)$. There were no intergroup differences between EPI and NE at each measurement point.

\section{Discussion}

In this study, a single bolus of esmolol was unable to attenuate the BIS and NE increases due to tracheal intubation during desflurane anesthesia, although the BIS value in the esmolol-1 
A

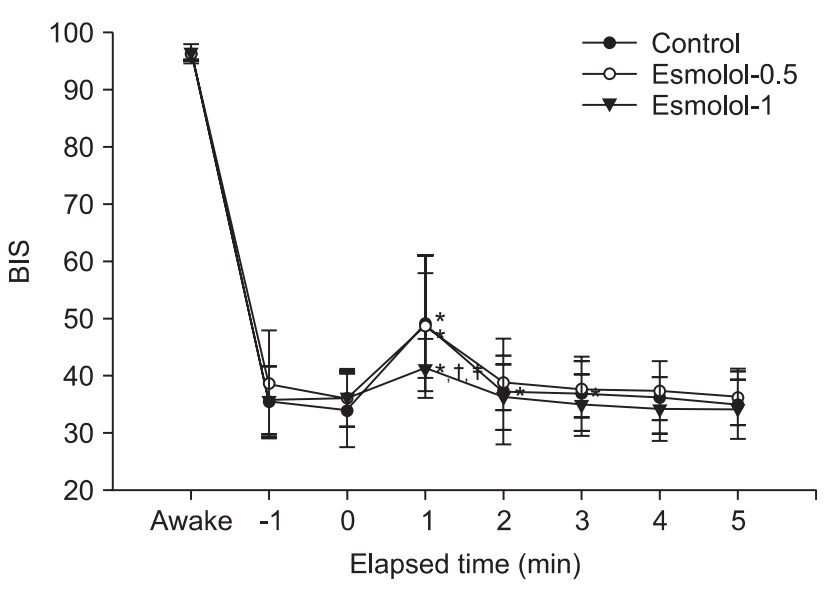

C

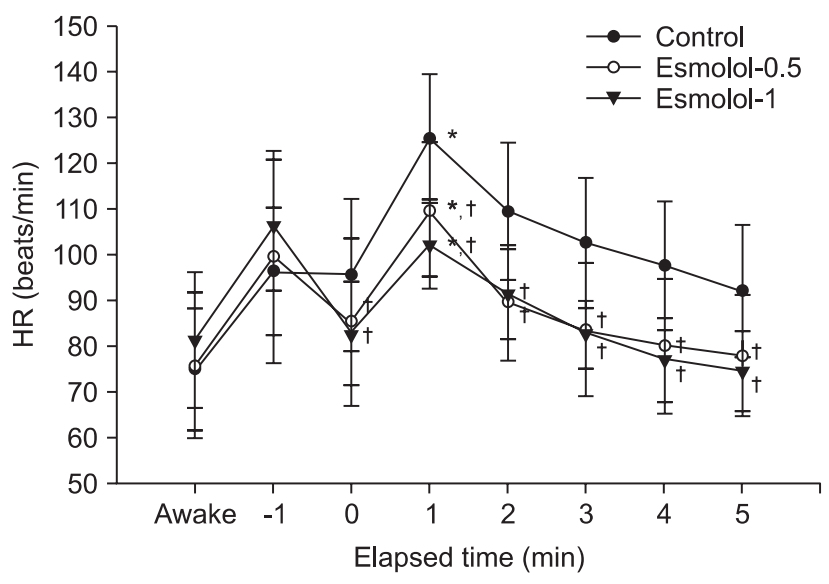

A

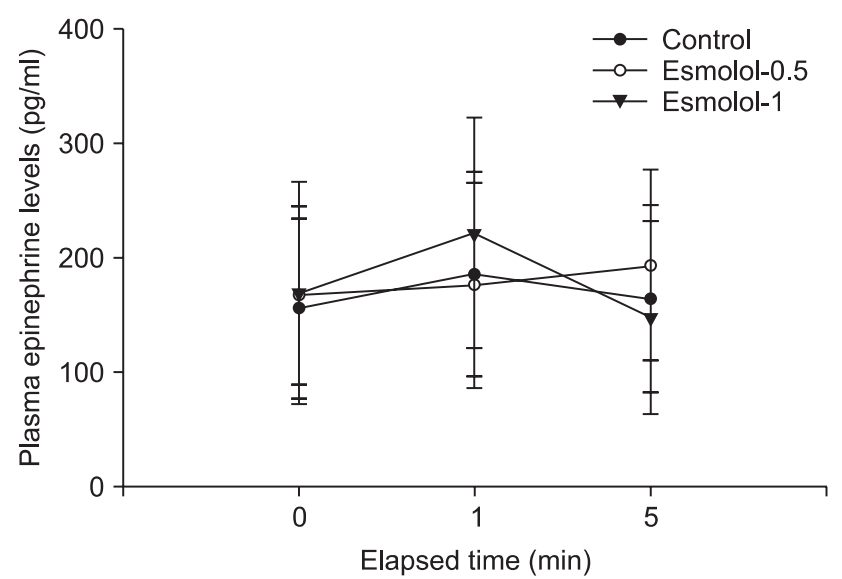

B

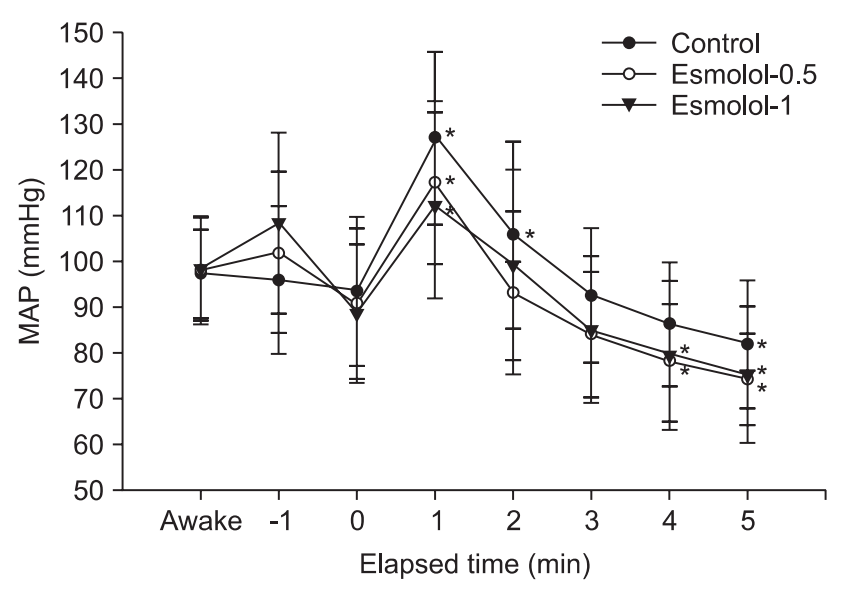

Fig. 2. Changes in BIS (A), MAP (B), and HR (C) before and after tracheal intubation in the three groups. Values are presented as mean $\pm \mathrm{SD}$. ${ }^{*} \mathrm{P}<0.05$ when compared to preintubation value. ${ }^{\dagger} \mathrm{P}<$ 0.05 when compared with the values at the same time point in the control group. ${ }^{\ddagger} \mathrm{P}<0.05$ esmolol-1 group when compared to the value in the esmolol-0.5 group at the same time point. -1 : before esmolol (normal saline) administration, 0: preintubation, 1 to $5: 1$ to 5 min after intubation. BIS: bispectral index, MAP: mean arterial pressure, HR: heart rate.

Fig. 3. Changes in epinephrine (A), and norepinephrine (B) before and after tracheal intubation in all three groups. Values are presented as mean $\pm \mathrm{SD}$. $* \mathrm{P}<0.05$ versus preintubation value. 0: preintubation, 1, 5: 1 and $5 \mathrm{~min}$ after intubation. 
group was lower than the other groups. The hemodynamic responses to laryngoscopic stimulation were similarly not blunted.

Previous studies have reported that anesthetic induction with desflurane increases cardiovascular responses via sympathetic activation $[8,9]$ and these responses could be blunted by drugs that decrease or block sympathetic outflow or activity, most notably opioids, $\beta$-adrenergic antagonists, and $\alpha_{2}$-adrenergic agonists [10].

The change in plasma catecholamine concentrations during desflurane anesthesia is more prominent than with other inhaled anesthetics due to reflex sympathetic activation from rapid increases in anesthetic concentration or laryngoscopic stimulation [7,9]. Esmolol could exert its effect on BIS by influencing sympathetic activation [2-5]. However, in the current study plasma NE concentrations 1 min after esmolol administration were not different among the three groups. Similarly, the BIS did not decrease to a greater degree in the esmolol groups prior to tracheal intubation. This observation is consistent with Berkenstadt's report [11], though in that study propofol with fentanyl was used during anesthesia induction and maintenance. As such, we contend that the lack of an effect of esmolol on BIS and serum catecholamine concentrations during desflurane anesthesia may relate to the esmolol regimen used.

Moreover, the plasma NE concentration in the esmolol group increased significantly following endotracheal intubation. Such an increase after the administration of esmolol has been reported in two settings: in individuals undergoing rapid sequence induction [12] and in patients given a single bolus of esmolol prior to intubation during isoflurane anesthesia [13]. Alterations in NE clearance after $\beta$-blockade, or greater baroreflex-mediated inhibition of the vasomotor center activity following pressor response in the control group was assumed to be one of the reasons [13]. Such increases in plasma NE concentration after a single bolus of esmolol have not been associated with desflurane anesthesia, but rather relate to the esmolol regimen. Because activation of the peripheral nerves via tracheal intubation is associated with increases in both BIS and circulating $\mathrm{NE}$ [1], it is unlikely that the administration of a single bolus could attenuate these sequelae. Here, sympathetic activation after laryngoscopic stimulation mediated by elevations in NE persisted despite the blunting of potential hemodynamic responses by opioids or $\beta$-adrenergic antagonists during desflurane anesthesia [7]. As such, regimens consisting of a loading dose followed by continuous infusion or simply continuous infusion of these drugs has been suggested as more efficacious in cases where the sympathetic system is substantially activated [3-4,14].

In our study, a single bolus administration of esmolol was unable to attenuate the hemodynamic responses to endo- tracheal intubation, with MAP and HR values 1 min after intubation having increased from preintubation values. Single bolus administrations of esmolol have proven insufficient in suppressing immediate hemodynamic response to laryngoscopic stimulation. One previous study demonstrated 11 different regimens and doses of esmolol as effective in attenuating HR and BP after tracheal intubation in a dose-dependent manner [14].

Notably, there are some key limitations in the study described here. First, plasma NE concentrations were not measured prior to the administration of esmolol during anesthesia induction. Nevertheless, as concentrations of NE did not differ at $1 \mathrm{~min}$ post-esmolol administration, esmolol is not believed to affect plasma catecholamine concentrations during desflurane anesthesia. Second, while BIS can be influenced by several factors, including changes in cardiac output that influence the pharmacokinetics of anesthetics [15], alveolar concentrations of poorly soluble drugs such as desflurane are the least sensitive to such changes.

In conclusion, our results demonstrate that a single bolus administration of esmolol is unable to attenuate BIS increases and catecholamine responses resulting from endotracheal intubation during desflurane anesthesia.

\section{References}

1. Guignard B, Menigaux C, Dupont X, Fletcher D, Chauvin M. The effect of remifentanil on the bispectral index change and hemodynamic responses after orotracheal intubation. Anesth Analg 2000; 90: 161-7.

2. Fernandez-Galinski S, Bermejo S, Mansilla R, Pol O, Puig MM. Comparative assessment of the effects of alfentanil, esmolol or clonidine when used as adjuvants during induction of general anaesthesia. Eur J Anaesthesiol 2004; 21: 476-82.

3. Oda Y, Nishikawa K, Hase I, Asada A. The short-acting betal-adrenoreceptor antagonists esmolol and landiolol suppress the bispectral index response to tracheal intubation during sevoflurane anesthesia. Anesth Analg 2005; 100: 733-7.

4. Menigaux C, Guignard B, Adam F, Sessler DI, Joly V, Chauvin M. Esmolol prevents movement and attenuates the BIS response to orotracheal intubation. Br J Anaesth 2002; 89: 857-62.

5. Choi SH, Kim CS, Kim JH, Kim BS, Kim EM, Min KT. A single dose of esmolol blunts the increase in bispectral index to tracheal intubation during sevoflurane but not desflurane anesthesia. J Neurosurg Anesthesiol 2009; 21: 214-7.

6. Ebert TJ, Muzi M, Lopatka CW. Neurocirculatory responses to sevoflurane in humans. A comparison to desflurane. Anesthesiology 1995; 83: 88-95.

7. Leung JM, Pastor DA. Dissociation between haemodynamics and sympathetic activation during anaesthetic induction with desfluranes. Can J Anaesth 1998; 45: 533-40.

8. Helman JD, Leung JM, Bellows WH, Pineda N, Roach GW, Reeves JD 3rd, et al. The risk of myocardial ischemia in patients receiving 
desflurane versus sufentanil anesthesia for coronary artery bypass graft surgery. The S.P.I. Research Group. Anesthesiology 1992; 77: 47-62.

9. Weiskopf RB, Moore MA, Eger EI 2nd, Noorani M, McKay L, Chortkoff B, et al. Rapid increase in desflurane concentration is associated with greater transient cardiovascular stimulation than with rapid increase in isoflurane concentration in humans. Anesthesiology 1994; 80: 1035-45.

10. Weiskopf RB, Eger EI 2nd, Noorani M, Daniel M. Fentanyl, esmolol, and clonidine blunt the transient cardiovascular stimulation induced by desflurane in humans. Anesthesiology 1994; 81: 1350-5.

11. Berkenstadt H, Loebstein R, Faibishenko I, Halkin H, Keidan I, Perel A. Effect of a single dose of esmolol on the bispectral index scale (BIS) during propofol/ fentanyl anaesthesia. Br J Anaesth 2002; 89: 509-11.
12. Ebert TJ, Bernstein JS, Stowe DF, Roerig D, Kampine JP. Attenuation of hemodynamic responses to rapid sequence induction and intubation in healthy patients with a single bolus of esmolol. J Clin Anesth 1990; 2: 243-52.

13. Thompson JP, West KJ, Hill AJ. The cardiovascular responses to double lumen endobronchial intubation and the effect of esmolol. Anaesthesia 1997; 52: 790-4.

14. Figueredo E, Garcia-Fuentes EM. Assessment of the efficacy of esmolol on the haemodynamic changes induced by laryngoscopy and tracheal intubation: a meta-analysis. Acta Anaesthesiol Scand 2001; 45: 1011-22.

15. Kurita T, Morita K, Kazama T, Sato S. Influence of cardiac output on plasma propofol concentrations during constant infusion in swine. Anesthesiology 2002; 96: 1498-503. 